\title{
IMA Commission on New Minerals, Nomenclature and Classification (CNMNC) - Newsletter 56
}

\author{
Ritsuro Miyawaki ${ }^{1}$, Frédéric Hatert ${ }^{2}$, Marco Pasero ${ }^{3}$, and Stuart J. Mills ${ }^{4}$ \\ ${ }^{1}$ Chairman, CNMNC I Department of Geology, National Museum of Nature and Science, \\ 4-1-1 Amakubo, Tsukuba 305-0005, Japan \\ ${ }^{2}$ Vice-Chairman, CNMNC I Laboratoire de Minéralogie, Université de Liège, \\ Bâtiment B18, Sart Tilman, 4000 Liège, Belgium \\ ${ }^{3}$ Vice-Chairman, CNMNC I Dipartimento di Scienze della Terra, Università di Pisa, \\ Via Santa Maria 53, 56126 Pisa, Italy \\ ${ }^{4}$ Secretary, CNMNC I Geosciences, Museum Victoria, P.O. Box 666, \\ Melbourne, Victoria 3001, Australia
}

Correspondence: Marco Pasero (marco.pasero@unipi.it)

Published: 6 August 2020

The information given here is provided by the IMA Commission on New Minerals, Nomenclature and Classification for comparative purposes and as a service to mineralogists working on new species.

Each mineral is described in the following format:

- mineral name, if the authors agree on its release prior to the full description appearing in press;

- chemical formula;

- type locality;

- full authorship of proposal;

- e-mail address of corresponding author;

- relationship to other minerals;

- crystal system, space group, structure determined, yes or no;

- unit-cell parameters;

- strongest lines in the X-ray powder diffraction pattern;

- type specimen repository and specimen number;

- citation details for the mineral prior to publication of full description.
Citation details concern the fact that this information will be published in the European Journal of Mineralogy on a routine basis, as well as being added month by month to the commission's web site.

It is still a requirement for the authors to publish a full description of the new mineral.

No other information will be released by the commission.

\section{New mineral proposals approved in June 2020}

IMA no. 2018-122a

Zinconigerite- $6 \mathrm{~N} 6 \mathrm{~S}$

$\mathrm{Zn}_{3} \mathrm{Sn}_{2} \mathrm{Al}_{16} \mathrm{O}_{30}(\mathrm{OH})_{2}$

Xianghualing skarn, Linwu County, Hunan Province, China $\left(25^{\circ} 26^{\prime} \mathrm{N}, 112^{\circ} 34^{\prime} \mathrm{E}\right)$

Can Rao*, Xiangping Gu, Rucheng Wang, Qunke Xia, Frédéric Hatert, and Fabrice Dal Bo

*E-mail: canrao@zju.edu.cn

Högbomite supergroup

Trigonal: $R \overline{3} m$; structure determined

$a=5.7241(2), c=55.539(2) \AA$

2.436(100), 1.931(100), 1.650(100), 1.646(100), 1.553(62), $1.430(61), 0.935(41), 0.911(40)$

Type material is deposited in the mineralogical collections of the Geological Museum of China, no. 16 Yangrou Hutong, Xisi, Beijing 100031, People's Republic of China, catalogue number M13811 
How to cite: Rao, C., Gu, X., Wang, R., Xia, Q., Hatert, F., and Dal Bo, F.: Zinconigerite-6N6S, IMA 2018122a, in: CNMNC Newsletter 56, Eur. J. Mineral., 32, https://doi.org/10.5194/ejm-32-443-2020, 2020.

\section{IMA no. 2020-009}

Gungerite

$\mathrm{TlAs}_{5} \mathrm{Sb}_{4} \mathrm{~S}_{13}$

Vorontsovskoe gold deposit, ca. $13 \mathrm{~km} \mathrm{~S}$ of the city of Krasnoturinsk, Sverdlovskaya Oblast, Northern Urals, Russia $\left(59^{\circ} 38^{\prime} 52^{\prime \prime} \mathrm{N}, 60^{\circ} 12^{\prime} 55^{\prime \prime} \mathrm{E}\right)$

Anatoly V. Kasatkin*, Jakub Plášil, Emil Makovicky, Nikita V. Chukanov, Radek Škoda, Atali A. Agakhanov, and Mikhail V. Tsyganko

*E-mail: anatoly.kasatkin@gmail.com

New structure type

Orthorhombic: $P b c n$; structure determined

$a=20.1958(3), b=11.5258(2), c=20.1430(2) \AA$

$5.755(100), \quad 4.273(4), \quad 3.705(6), \quad 3.030(10), \quad 2.901(10)$, $2.878(14), 2.850(5), 2.821(10)$

Type material is deposited in the collections of the Fersman Mineralogical Museum, Russian Academy of Sciences, Leninskiy Prospekt 18-2, Moscow 119071, Russia, registration number 5518/1

How to cite: Kasatkin, A. V., Plášil, J., Makovicky, E., Chukanov, N. V., Škoda, R., Agakhanov, A. A., and Tsyganko, M.V.: Gungerite, IMA 2020-009, in: CNMNC Newsletter 56, Eur. J. Mineral., 32, https://doi.org/10.5194/ejm-32-443-2020, 2020.

\section{IMA no. 2020-010}

Muonionalustaite

$\mathrm{Ni}_{3}(\mathrm{OH})_{4} \mathrm{Cl}_{2} \cdot 4 \mathrm{H}_{2} \mathrm{O}$

On the corrosion crust of the Muonionalusta iron meteorite, Pajala, ca. $1.5 \mathrm{~km}$ NE of lake Kitkiöjärvi, Norrbotten, Swedish Lapland, Sweden $\left(67^{\circ} 50^{\prime} 25^{\prime \prime} \mathrm{N}, 23^{\circ} 11^{\prime} 00^{\prime \prime} \mathrm{E}\right.$, 254 m a.s.1.)

Dan Holtstam*, Luca Bindi, Andreas Karlsson, Johan Söderhielm, and Anders Zetterqvist

*E-mail: dan.holtstam@nrm.se

Known synthetic analogue

Monoclinic: $C 2 / m$; structure determined

$a=15.018(3), \quad b=3.1490(6), \quad c=10.502(3) \AA$,

$\beta=101.535(15)^{\circ}$

10.30(100), $7.36(30), \quad 5.49(67), \quad 3.868(31), \quad 2.917(14)$, 2.692(21), 2.409(25), 2.245(21)

Type material is deposited in the mineralogical collections of the Department of Geosciences, Swedish Museum of Natural History, Box 50007, SE-10405 Stockholm, Sweden, collection numbers GEO-NRM nos. 20050144 and 20050145

How to cite: Holtstam, D., Bindi, L., Karlsson, A., Söderhielm, J., and Zetterqvist, A.: Muonionalustaite, IMA 2020-
010, in: CNMNC Newsletter 56, Eur. J. Mineral., 32, https://doi.org/10.5194/ejm-32-443-2020, 2020.

\section{IMA no. 2020-011}

Calcioveatchite

$\mathrm{SrCaB}_{11} \mathrm{O}_{16}(\mathrm{OH})_{5} \cdot \mathrm{H}_{2} \mathrm{O}$

Nepskoe potassium salt deposit (drill core), Nepa River basin, Lower Tunguska, Irkutsk Oblast, Siberia, Russia $\left(59^{\circ} 01^{\prime} 48^{\prime \prime} \mathrm{N}, 108^{\circ} 00^{\prime} 00^{\prime \prime} \mathrm{E}\right)$

Igor V. Pekov*, Natalia V. Zubkova, Vladimir N. Apollonov, Vasiliy O. Yapaskurt, Nikita V. Chukanov, Sergey N. Britvin, and Dmitry Y. Pushcharovsky

*E-mail: igorpekov@mail.ru

The $\mathrm{Sr} / \mathrm{Ca}$-ordered analogue of veatchite

Monoclinic: $P 2_{1}$; structure determined

$a=6.7030(3), \quad b=20.6438(9), \quad c=6.6056(3) \AA$

$\beta=119.153(7)^{\circ}$

10.35(100), 5.633(12), 5.092(10), 3.447(14), 3.362(13), $3.309(38), 2.862(10), 2.585(19)$

Type material is deposited in the collections of the Fersman Mineralogical Museum, Russian Academy of Sciences, Leninskiy Prospekt 18-2, Moscow 119071, Russia, registration number 5482/1

How to cite: Pekov, I. V., Zubkova, N. V., Apollonov, V. N., Yapaskurt, V. O., Chukanov, N. V., Britvin, S. N., and Pushcharovsky, D. Y.: Calcioveatchite, IMA 2020011, in: CNMNC Newsletter 56, Eur. J. Mineral., 32, https://doi.org/10.5194/ejm-32-443-2020, 2020.

\section{IMA no. 2020-013}

\section{Svetlanaite}

$\mathrm{SnSe}$

In quartz veins of the Ozernovskoe deposit, Kamchatka Peninsula, Russia $\left(57^{\circ} 35^{\prime} 36^{\prime \prime} \mathrm{N}, 160^{\circ} 50^{\prime} 60^{\prime \prime} \mathrm{E}\right)$

Victor M. Okrugin, Anna Vymazalová*, Vladimir V. Kozlov, František Laufek, Chris J. Stanley, and Ilya Shkilsky

*E-mail: anna.vymazalova@geology.cz

Known synthetic analogue

Orthorhombic: Pnma

$a=11.52, b=4.16, c=4.44 \AA$

3.517(4), 2.934(7), 2.875(100), 2.378(7), 2.086(5), 2.043(4), $1.833(10), 1.437(9)$

Type material is deposited in the mineralogical collections of the Department of Earth Sciences, Natural History Museum, Cromwell Road, London, SW7 5BD, United Kingdom, catalogue number BM 2020,2

How to cite: Okrugin, V. M., Vymazalová, A., Kozlov, V. V., Laufek, F., Stanley, C. J., and Shkilsky, I.: Svetlanaite, IMA 2020-013, in: CNMNC Newsletter 56, Eur. J. Mineral., 32, https://doi.org/10.5194/ejm-32-443-2020, 2020. 
IMA no. 2020-015

Saranovskite-(Y)

$\mathrm{SrCaFe}_{2}^{2+}\left(\mathrm{Cr}_{4} \mathrm{Ti}_{2}\right) \mathrm{Ti}_{12} \mathrm{O}_{38}$

Rudnaya underground chromite mine (depth $400 \mathrm{~m}$ ), Glavnoe Saranovskoe deposit, Sarany town, $5 \mathrm{~km} \mathrm{~N}$ of the railway station Laki, Gornozavodskiy district, Permskiy Kray, Middle Urals, Russia $\left(58^{\circ} 30^{\prime} \mathrm{N}, 58^{\circ} 52^{\prime} \mathrm{E}\right)$

Nikita V. Chukanov*, Ramiza K. Rastsvetaeva, Oleg K. Ivanov, Olga N. Kazheva, Igor V. Pekov, Atali A. Agakhanov, Konstantin V. Van, and Sergey N. Britvin

*E-mail: nikchukanov@yandex.ru

Crichtonite group

Trigonal: $R \overline{3}$; structure determined

$a=10.3553(2), c=20.7301(4) \AA$

$3.398(75), 3.052(48), 2.881(100), 2.842(65), 2.247(67)$, 2.137(76), 1.597(72), 1.439(76)

Type material is deposited in the collections of the Fersman Mineralogical Museum, Russian Academy of Sciences, Leninskiy Prospekt 18-2, Moscow 119071, Russia, registration number 5558/1

How to cite: Chukanov, N. V., Rastsvetaeva, R. K., Ivanov, O. K., Kazheva, O. N., Pekov, I. V., Agakhanov, A. A., Van, K. V., and Britvin, S. N.: Saranovskite, IMA 2020015, in: CNMNC Newsletter 56, Eur. J. Mineral., 32, https://doi.org/10.5194/ejm-32-443-2020, 2020.

\section{IMA no. 2020-017}

Strontioborite

$\mathrm{Sr}\left[\mathrm{B}_{8} \mathrm{O}_{11}(\mathrm{OH})_{4}\right]$

In cores of boreholes drilled at the Chelkar salt dome, near Chelkar (Shalkar) lake, Kazakhstan

Igor V. Pekov*, Natalia V. Zubkova, Nikita V. Chukanov, Vasiliy O. Yapaskurt, Sergey N. Britvin, and Dmitry Y. Pushcharovsky

*E-mail: igorpekov@mail.ru

New structure type

Monoclinic: $P 2_{1}$; structure determined

$a=7.6192(3), \quad b=8.1867(2), \quad c=9.9164(3) \AA$,

$\beta=108.357(4)^{\circ}$

$7.22(100), \quad 5.409(61), \quad 4.090(64), 3.300(48), \quad 3.082(28)$, 2.926(25), 2.121(30), 2.043(37)

Type material is deposited in the collections of the Fersman Mineralogical Museum, Russian Academy of Sciences, Leninskiy Prospekt 18-2, Moscow 119071, Russia, registration numbers 69851 (holotype) and ST-7069 (cotype)

How to cite: Pekov, I. V., Zubkova, N. V., Chukanov, N. V., Yapaskurt, V. O., Britvin, S. N., and Pushcharovsky, D. Y.: Strontioborite, IMA 2020-017, in: CNMNC Newsletter 56, Eur. J. Mineral., 32, https://doi.org/10.5194/ejm-32-4432020, 2020.

\section{IMA no. 2020-019}

Wildcatite

$\mathrm{CaFe}^{3+} \mathrm{Te}^{6+} \mathrm{O}_{5}(\mathrm{OH})$

Wildcat prospect ("high grade hill"), Detroit Mining District, Juab Co., Utah, USA ( $\left.39^{\circ} 35^{\prime} 30^{\prime \prime} \mathrm{N}, 113^{\circ} 06^{\prime} 57^{\prime \prime} \mathrm{W}\right)$

Owen P. Missen, Stuart J. Mills*, Anthony R. Kampf, Mark F. Coolbaugh, Jens Najorka, Joe Marty, John Spratt, Mati Raudsepp, John K. McCormack, and Mike S. Rumsey

*E-mail: smills@museum.vic.gov.au

New structure type

Trigonal: $P \overline{3} 1 \mathrm{~m}$; structure determined

$a=5.200(1), c=4.967(1) \AA$

4.977(31), 3.332(100), 2.597(55), 2.301(59), 2.050(33), 1.795(88), 1.610(30), 1.499(30)

Cotype material is deposited in the mineralogical collections of the Natural History Museum of Los Angeles County, 900 Exposition Boulevard, Los Angeles, CA 90007, USA, specimen numbers 74538, 74539, and 74540; the Museums Victoria, GPO Box 666, Melbourne, Victoria 3001, Australia, specimen numbers M55257 and M55258; the Natural History Museum, Cromwell Road, London SW7 5BD, United Kingdom, specimen number BM 2020,4; and the W. M. Keck Earth Science and Mineral Engineering Museum, University of Nevada, 1664 N. Virginia Street, Reno, NV 89557, USA, specimen number 2020.002.001

How to cite: Missen, O. P., Mills, S. J., Kampf, A. R., Coolbaugh, M. F., Najorka, J., Marty, J., Spratt, J., Raudsepp, M., McCormack, J. K., and Rumsey, M. S.: Wildcatite, IMA 2020-019, in: CNMNC Newsletter 56, Eur. J. Mineral., 32, https://doi.org/10.5194/ejm-32-443-2020, 2020.

\section{IMA no. 2020-020}

Biraite-(La)

$\mathrm{La}_{2} \mathrm{Fe}^{2+}\left(\mathrm{CO}_{3}\right)\left(\mathrm{Si}_{2} \mathrm{O}_{7}\right)$

Mochalin Log REE deposit, $14 \mathrm{~km} \mathrm{~N}$ of the city of Kyshtym, Chelyabinsk Oblast, South Urals, Russia $\left(55^{\circ} 48^{\prime} 42^{\prime \prime} \mathrm{N}\right.$, $60^{\circ} 33^{\prime} 46^{\prime \prime} \mathrm{E}$ )

Anatoly V. Kasatkin*, Natalia V. Zubkova, Igor V. Pekov, Nikita V. Chukanov, Radek Škoda, Atali A. Agakhanov, Dmitriy I. Belakovskiy, Aleksey M. Kuznetsov, and Dmitry Y. Pushcharovsky

*E-mail: anatoly.kasatkin@gmail.com

Biraite group

Monoclinic: $P 2_{1} / c$; structure determined

$a=6.566(1), \quad b=6.767(1), \quad c=18.698(3) \AA, \quad \beta=$ $108.95(2)^{\circ}$

4.594(49), 3.723(47), 3.055(100), 2.962(66), 2.787(35), 2.690(38), 2.509(26), 1.843(33)

Type material is deposited in the collections of the Fersman Mineralogical Museum, Russian Academy of Sciences, Leninskiy Prospekt 18-2, Moscow 119071, Russia, registration number 5557/1 
How to cite: Kasatkin, A. V., Zubkova, N. V., Pekov, I. V., Chukanov, N. V., Škoda, R., Agakhanov, A. A., Belakovskiy, D. I., Kuznetsov, A. M., and Pushcharovsky, D. Y.: Biraite-(La), IMA 2020-020, in: CNMNC Newsletter 56, Eur. J. Mineral., 32, https://doi.org/10.5194/ejm-32-4432020, 2020 .

\section{IMA no. 2020-021}

Ferro-papikeite $\mathrm{NaFe}_{2}^{2+}\left(\mathrm{Fe}_{3}^{2+} \mathrm{Al}_{2}\right)\left(\mathrm{Si}_{5} \mathrm{Al}_{3}\right) \mathrm{O}_{22}(\mathrm{OH})_{2}$

Filipstad Municipality, Värmland County, Nordmark, Swe$\operatorname{den}\left(59^{\circ} 50^{\prime} 37.53^{\prime \prime} \mathrm{N}, 14^{\circ} 06^{\prime} 3.49^{\prime \prime} \mathrm{E}\right)$

Frank C. Hawthorne*, Maxwell C. Day, Kees Linthout, Wim J. Lustenhouwer, and Roberta Oberti

*E-mail: frank_hawthorne@umanitoba.ca

Amphibole supergroup

Orthorhombic: Pnma; structure determined

$a=18.628(4), b=17.888(4), c=5.303(1) \AA$

$8.255(99), \quad 3.223(39), \quad 3.056(68), 2.674(41), \quad 2.572(56)$, 2.549(38), 2.501(50), 1.991(31)

Type material is deposited in the mineralogical collections of the Royal Ontario Museum, 100 Queens Park, Toronto, ON M5S 2C6, Canada, accession number M60100

How to cite: Hawthorne, F. C., Day, M. C., Linthout, K., Lustenhouwer, W. J., and Oberti, R.: Ferro-papikeite, IMA 2020-021, in: CNMNC Newsletter 56, Eur. J. Mineral., 32, https://doi.org/10.5194/ejm-32-443-2020, 2020.

\section{New mineral proposals approved in July 2020}

\section{IMA no. 2020-023}

Kishonite

$\mathrm{VH}_{2}$

Kishon Mid Reach zone 2, Kishon river, Haifa district, Israel Luca Bindi*, Fernando Cámara, William L. Griffin, JinXiang Huang, Sarah E.M. Gain, Vered Toledo, and Suzanne Y. O'Reilly

*E-mail: luca.bindi@unifi.it

Known synthetic analogue

Cubic: $F m \overline{3} m$; structure determined

$a=4.268(1) \AA$

$2.464(100), \quad 2.134(42), \quad 1.509(27), \quad 1.287(26), \quad 1.232(7)$, 0.979(10), 0.954(10), 0.871(10)

Type material is deposited in the mineralogical collections of the Museo di Storia Naturale, Università di Firenze, Via G. La Pira 4, I-50121, Florence, Italy, catalogue number 3364/I How to cite: Bindi, L., Cámara, F., Griffin, W. L., Huang, J.X., Gain, S. E. M., Toledo, V., and O'Reilly, S. Y.: Kishonite, IMA 2020-023, in: CNMNC Newsletter 56, Eur. J. Mineral., 32, https://doi.org/10.5194/ejm-32-443-2020, 2020.

\section{IMA no. 2020-024}

Shakhdaraite-(Y)

$\mathrm{ScYNb}_{2} \mathrm{O}_{8}$

Leskhozovskiy granitic pegmatite, right side of the Shakhdara river, a left tributary of the Gunt River, southwestern Pamir, Roshtkala district, Gorno-Badakhshan $\mathrm{Au}$ tonomous Region, Tajikistan $\left(37^{\circ} 11^{\prime} 43^{\prime \prime} \mathrm{N}, 71^{\circ} 52^{\prime} 04^{\prime \prime} \mathrm{E}\right)$

Leonid A. Pautov*, Mirak A. Mirakov, Elena Sokolova, Maxwell C. Day, Frank C. Hawthorne, Manuchekhr A. Shodibekov, Vladimir Y. Karpenko, Saimudasir Makhmadsharif, and Abdulkhak R. Faiziev

*E-mail: pla58@mail.ru

Structurally related to brookite

Monoclinic: $P 2 / c$; structure determined

$a=9.930(2), b=5.662(1), c=5.211(1) \AA, \beta=92.38(3)^{\circ}$

$3.72(35), 3.073(100), 2.990(85), 2.832(20), 2.603(24)$, 2.484(33), 1.916(23), 1.867(21)

Type material is deposited in the collections of the Fersman Mineralogical Museum, Russian Academy of Sciences, Leninskiy Prospekt 18-2, Moscow 119071, Russia, registration number 5569/1

How to cite: Pautov, L. A., Mirakov, M. A., Sokolova, E., Day, M. C., Hawthorne, F. C., Shodibekov, M. A., Karpenko, V. Y., Makhmadsharif, S., and Faiziev, A. R.: Shakhdaraite(Y), IMA 2020-024, in: CNMNC Newsletter 56, Eur. J. Mineral., 32, https://doi.org/10.5194/ejm-32-443-2020, 2020.

\section{IMA no. 2020-025}

Puttapaite

$\mathrm{Pb}_{2} \mathrm{Mn}_{2}^{2+} \mathrm{ZnCr}_{4}^{3+} \mathrm{O}_{2}\left(\mathrm{AsO}_{4}\right)_{4}(\mathrm{OH})_{6} \cdot 12 \mathrm{H}_{2} \mathrm{O}$

Beltana deposit, Puttapa, Flinders Ranges, South Australia, Australia $\left(30^{\circ} 40^{\prime} 32^{\prime \prime} \mathrm{S}, 138^{\circ} 26^{\prime} 04^{\prime \prime} \mathrm{E}\right)$

Peter Elliott* and Anthony R. Kampf

*E-mail: peter.elliott@adelaide.edu.au

New structure type

Monoclinic: $C 2 / m$; structure determined

$a=12.405(3), \quad b=10.565(2), \quad c=12.311(3) \AA$,

$\beta=106.06(3)^{\circ}$

11.74(100), 7.88(23), 5.223(40), 4.797(48), 3.958(44), 3.605(71), 3.013(20), 2.686(32)

Type material is deposited in the mineralogical collections of the South Australian Museum, North Terrace, Adelaide, South Australia 5000, Australia, registration number G34869 How to cite: Elliott, P. and Kampf, A. R.: Puttapaite, IMA 2020-025, in: CNMNC Newsletter 56, Eur. J. Mineral., 32, https://doi.org/10.5194/ejm-32-443-2020, 2020. 
IMA no. 2020-026

Anastasenkoite $\mathrm{CaFe}^{2+} \mathrm{P}_{2} \mathrm{O}_{7}$

Halamish wadi (Nahal Halamish), Hatrurim Basin, Negev desert, Israel $\left(31^{\circ} 09^{\prime} 47^{\prime \prime} \mathrm{N}, 35^{\circ} 17^{\prime} 57^{\prime \prime} \mathrm{E}\right)$

Sergey N. Britvin*, Michail N. Murashko, Yevgeny Vapnik, Natalia S. Vlasenko, Oleg S. Vereshchagin, Vladimir N. Bocharov, Maria G. Krzhizhanovskaya, Maksim S. Lozhkin, and Anatoly A. Zolotarev

*E-mail: sbritvin@gmail.com

Known synthetic analogue

Triclinic: $P \overline{1} ;$ structure determined

$a=6.576(1), b=6.541(1), c=6.547(1) \AA, \alpha=67.11(1)$,

$\beta=82.35(1), \gamma=87.27(2)^{\circ}$

5.421(10), 3.222(29), 3.057(9), 3.052(41), 3.013(52), 2.866(100), 2.600(14), 2.159(10)

Type material is deposited in the collections of the Fersman Mineralogical Museum, Russian Academy of Sciences, Leninskiy Prospekt 18-2, Moscow 119071, Russia, registration number 5289/1

How to cite: Britvin, S. N., Murashko, M. N., Vapnik, Y., Vlasenko, N. S., Vereshchagin, O. S., Bocharov, V. N., Krzhizhanovskaya, M. G., Lozhkin, M. S., and Zolotarev, A. A.: Anastasenkoite, IMA 2020-026, in: CNMNC Newsletter 56, Eur. J. Mineral., 32, https://doi.org/10.5194/ejm-32-4432020, 2020.

\section{IMA no. 2020-027}

Selenolaurite

$\mathrm{RuSe}_{2}$

Ingul gold-bearing placer, Nepryakhino placer zone, $25 \mathrm{~km}$ ENE of Miass, Chelyabinsk Oblast, Russia $\left(55^{\circ} 04^{\prime} 49^{\prime \prime} \mathrm{N}\right.$, $60^{\circ} 34^{\prime} 11^{\prime \prime} \mathrm{E}$ )

Elena V. Belogub, Sergey N. Britvin, Vladimir V. Shilovskikh, Leonid A. Pautov, Vasiliy A. Kotlyarov, Maria G. Krzhizhanovskaya, Konstantin A. Novoselov, Elisaveta V. Zaykova, and Ivan A. Blinov

*E-mail: belogub_e@yahoo.com

The Se analogue of laurite

Cubic: $P a \overline{3}$; structure determined

$a=5.9424(2) \AA$

3.434(41), 2.973(90), 2.658(100), 2.426(84), 1.791(87), 1.715(12), 1.648(18), 1.328(8)

Type material is deposited in the mineralogical collections of the Natural Scientific Museum of the Ilmen State Reserve, Miass 456317, Russia, registration number 17503

How to cite: Belogub, E. V., Britvin, S. N., Shilovskikh, V. V., Pautov, L. A., Kotlyarov, V. A., Krzhizhanovskaya, M. G., Novoselov, K. A., Zaykova, E. V., and Blinov, I. A.: Selenolaurite, IMA 2020-027, in: CNMNC Newsletter 56, Eur. J. Mineral., 32, https://doi.org/10.5194/ejm-32-4432020, 2020.

\section{IMA no. 2020-028}

Monteneroite

$\mathrm{Cu}^{2+} \mathrm{Mn}_{2}^{2+}\left(\mathrm{AsO}_{4}\right)_{2} \cdot 8 \mathrm{H}_{2} \mathrm{O}$

Monte Nero mine, Rocchetta di Vara, La Spezia, Liguria, Italy $\left(44^{\circ} 14^{\prime} 48^{\prime \prime} \mathrm{N}, 9^{\circ} 45^{\prime} 27^{\prime \prime} \mathrm{E}\right)$

Anthony R. Kampf*, Jakub Plášil, Barbara P. Nash, Marco

E. Ciriotti, Fabrizio Castellaro, and Luigi Chiappino

*E-mail: akampf@nhm.org

Structurally related to vivianite

Monoclinic: $C 2 / \mathrm{m}$; structure determined

$a=10.367(1), \quad b=13.713(2), \quad c=4.8420(8) \AA$,

$\beta=105.992(8)^{\circ}$

$8.08(38), \quad 6.86(100), \quad 4.507(50), \quad 3.287(75), \quad 3.032(94)$, 2.751(88), 2.372(44), 1.938(35)

Type material is deposited in the mineralogical collections of the Natural History Museum of Los Angeles County, 900 Exposition Boulevard, Los Angeles, CA 90007, USA, catalogue number 67509

How to cite: Kampf, A. R., Plášil, J., Nash, B. P., Ciriotti, M. E., Castellano, F., and Chiappino, L.: Monteneroite, IMA 2020-028, in: CNMNC Newsletter 56, Eur. J. Mineral., 32, https://doi.org/10.5194/ejm-32-443-2020, 2020.

\section{IMA no. 2020-029}

Magnesiohögbomite-6N12S

$\mathrm{Mg}_{5} \mathrm{Al}_{11} \mathrm{TiO}_{23}(\mathrm{OH})$

DeWitts Corners area, lot 10, concession 1, Bathurst Township, Lanark County, Ontario, Canada $\left(44^{\circ} 49^{\prime} 47^{\prime \prime} \mathrm{N}\right.$, $\left.76^{\circ} 21^{\prime} 05^{\prime \prime} \mathrm{W}\right)$

Inna Lykova*, Ralph Rowe, Glenn Poirier, Gerald Giester, and Kate Helwig

*E-mail: ilykova@nature.ca

Högbomite supergroup

Trigonal: $R \overline{3} m$; structure determined

$a=5.7194(2), c=83.069(5) \AA$

$2.921(26), 2.863(49), 2.687(29), 2.547(31), 2.434(100)$,

2.073(22), 1.988(23), 1.556(22)

Type material is deposited in the mineralogical collections of the Canadian Museum of Nature, 240 McLeod Street, Ottawa, Ontario, Canada, catalogue number CMNMC 87484

How to cite: Lykova, I., Rowe, R., Poirier, G., Giester, G., and Helwig, K.: Magnesiohögbomite-6N12S, IMA 2020029, in: CNMNC Newsletter 56, Eur. J. Mineral., 32, https://doi.org/10.5194/ejm-32-443-2020, 2020.

\section{IMA no. 2020-031}

Zinkgruvanite

$\mathrm{Ba}_{4} \mathrm{Mn}_{4}^{2+} \mathrm{Fe}_{2}^{3+}\left(\mathrm{Si}_{2} \mathrm{O}_{7}\right)_{2}\left(\mathrm{SO}_{4}\right)_{2} \mathrm{O}_{2}(\mathrm{OH})_{2}$

Zinkgruvan $\mathrm{Zn}-\mathrm{Pb}-\mathrm{Ag}$ deposit, near Åmmeberg, Askersund Municipality, Örebro County, Sweden $\left(58^{\circ} 49^{\prime} \mathrm{N}, 15^{\circ} 06^{\prime} \mathrm{E}\right)$ 
Fernando Cámara, Dan Holtstam*, Nils Jansson, Erik Jonsson, Andreas Karlsson, Jörgen Langhof, Jaroslaw Majka, and Anders Zetterqvist

*E-mail: dan.holtstam@nrm.se

Ericssonite group

Triclinic: $P \overline{1}$; structure determined

$a=5.3982(1), \quad b=7.0237(1), \quad c=14.8108(4) \AA$,

$\alpha=98.256(2), \beta=93.379(2), \gamma=89.985(2)^{\circ}$

$3.508(70), 2.980(70), 2.814(68), 2.777(70), 2.699(71)$, 2.680(85), 2.125(100), 2.107(96)

Type material is deposited in the mineralogical collections of the Department of Geosciences, Swedish Museum of Natural History, Box 50007, SE-10405 Stockholm, Sweden, collection number GEO-NRM no. 20170502

How to cite: Cámara, F., Holtstam, D., Jansson, N., Jonsson, E., Karlsson, A., Langhof, J., Majka, J., and Zetterqvist, A.: Zinkgruvanite, IMA 2020-031, in: CNMNC Newsletter 56, Eur. J. Mineral., 32, https://doi.org/10.5194/ejm-32-4432020, 2020.

\section{IMA no. 2020-032}

\section{Krupičkaite}

$\mathrm{Cu}_{6}\left[\mathrm{AsO}_{3}(\mathrm{OH})\right]_{6} \cdot 8 \mathrm{H}_{2} \mathrm{O}$

Geister vein, third Geister level of the Rovnost (Werner) mine, Jáchymov, Western Bohemia, Czech Republic $\left(50^{\circ} 22^{\prime} 08^{\prime \prime} \mathrm{N}, 12^{\circ} 53^{\prime} 49^{\prime \prime} \mathrm{E}\right)$

Gwladys Steciuk, Jakub Plášil*, Jiří Sejkora, Jiří Čejka, and Jan Hloušek

*E-mail: plasil@fzu.cz

Chemically and structurally close to geminite

Monoclinic: $P 2{ }_{1} / n$; structure determined

$a=15.504(7), \quad b=18.144(7), \quad c=10.563(5) \AA$,

$\beta=103.30(4)^{\circ}$

9.089(100), 4.394(0.5), 3.909(0.5), 3.533(1.0), 3.369(0.5), 3.244(0.4), 2.972(0.9), 2.937(0.5)

Type material is deposited in the mineralogical collections of the Department of Mineralogy and Petrology, National Museum in Prague, Cirkusová 1740, 19300 Prague 9, Czech Republic, catalogue number P1P 18/2020

How to cite: Steciuk, G., Plášil, J., Sejkora, J., Čejka, J., and Hloušek, J.: Krupičkaite, IMA 2020032, in: CNMNC Newsletter 56, Eur. J. Mineral., 32, https://doi.org/10.5194/ejm-32-443-2020, 2020.

\section{IMA no. 2020-004a}

Allantoin

$\mathrm{C}_{4} \mathrm{H}_{6} \mathrm{~N}_{4} \mathrm{O}_{3}$

Rowley mine 125-foot level, ca. $20 \mathrm{~km} \mathrm{NW}$ of Theba, Maricopa Co., Arizona, USA ( $\left.33^{\circ} 02^{\prime} 57^{\prime \prime} \mathrm{N}, 113^{\circ} 01^{\prime} 50^{\prime \prime} \mathrm{W}\right)$

Anthony R. Kampf*, Aaron J. Celestian, Barbara P. Nash, and Joe Marty
*E-mail: akampf@nhm.org

Known synthetic analogue

Monoclinic: $P 2_{1} / c$; structure determined

$a=8.0304(9), \quad b=5.1596(5), \quad c=14.801(2) \AA$,

$\beta=93.017(7)^{\circ}$

5.567(31), 4.864(37), 4.341(30), 4.197(100), 3.604(40),

$3.190(33), 3.010(51), 2.809(42)$

Type material is deposited in the mineralogical collections of the Natural History Museum of Los Angeles County, 900 Exposition Boulevard, Los Angeles, CA 90007, USA, catalogue number 74491

How to cite: Kampf, A. R., Celestian, A. J., Nash, B. P., and Marty, J.: Allantoin, IMA 2020-004a, in: CNMNC Newsletter 56, Eur. J. Mineral., 32, https://doi.org/10.5194/ejm-32443-2020, 2020.

\section{Nomenclature/classification proposals approved in July 2020}

IMA 20-C: Crystal structure and redefinition of utahite

Proposal 20-C is accepted, and the formula of utahite is redefined from $\mathrm{Cu}_{5}^{2+} \mathrm{Zn}_{3}\left(\mathrm{Te}^{6+} \mathrm{O}_{4}\right)_{4}(\mathrm{OH})_{8} \cdot 7 \mathrm{H}_{2} \mathrm{O}$ to $\mathrm{MgCu}_{4}^{2+} \mathrm{Zn}_{2} \mathrm{Te}_{3}^{6+} \mathrm{O}_{14}(\mathrm{OH})_{4} \cdot 6 \mathrm{H}_{2} \mathrm{O}$

\section{Margarosanite group}

The margarosanite group is established and includes the three isostructural members: margarosanite, walstromite, and breyite.

\section{Pascoite family}

The pascoite family is established and consists of $20 \mathrm{~V}$ minerals. The family consists of the pascoite and lasalite groups as well as a number of ungrouped members. In addition, the formula of rakovanite has been revised from $\mathrm{Na}_{3}\left[\mathrm{H}_{3} \mathrm{~V}_{10} \mathrm{O}_{28}\right] \cdot 15 \mathrm{H}_{2} \mathrm{O}$ to $\left(\mathrm{NH}_{4}\right)_{3} \mathrm{Na}_{3}\left[\mathrm{~V}_{10} \mathrm{O}_{28}\right] \cdot 12 \mathrm{H}_{2} \mathrm{O}$. 